\title{
The prognostic value of the lymphocyte-to-monocyte ratio for high-risk papillary thyroid carcinoma
}

This article was published in the following Dove Press journal:

Cancer Management and Research

\author{
Linlin Song $\mathbb{D}^{1,2}$ \\ Jingqiang Zhu' \\ Zhihui $\mathrm{Li}^{1,3}$ \\ Tao Wei ${ }^{1,3}$ \\ Rixiang Gong ${ }^{1,3}$ \\ Jianyong Lei ${ }^{1}$
}

'Thyroid and Parathyroid Surgery Center, West China Hospital of Sichuan University, Chengdu 61004I, People's Republic of China; ${ }^{2}$ West China School of Medicine, Sichuan University, Chengdu 610065, People's Republic of China; ${ }^{3}$ Thyroid and Breast Surgery Center, Chengdu Shang Jin Nan Fu Hospital, Chengdu 611700, People's Republic of China
Correspondence: Jianyong Lei

Thyroid and Parathyroid Surgery Center,

West China Hospital of Sichuan

University, Chengdu 61004I, People's

Republic of China

Tel +86 2885423822

Fax +86 2885423822

Email leijianyongI I@I63.com
Background and Aims: The prognosis of papillary thyroid carcinoma (PTC) is highly variable, even for high-risk cases. The predictive and prognostic role of the lymphocyte-tomonocyte ratio (LMR) has been reported in other cancers. The aim of our present study was to explore the value of LMR prognostic prediction in high-risk PTC patients.

Patients and Methods: Two hundred and twenty-four PTC high-risk cases at West China Hospital were randomized into a training set (112 cases) and testing set (112 cases), while 48 cases in Shang Jin Nan Fu Hospital were included as the external validation set.

Results: A lower preoperative LMR correlated with larger tumor size, advanced $\mathrm{N}$ and $\mathrm{M}$ stages, and an increased number of multiple PTC cases in the training, testing, and validation sets (all $P<0.05$ in the three sets). Patients with a high LMR exhibited significantly improved overall and PTC-free survival compared with those of patients with a low LMR in the training, testing, validation, and combined sets (all $P<0.05$ in the individual and combined sets). Moreover, multivariate analyses identified the LMR as an independent prognostic factor for overall and PTC-free survival. The nomograms for predicting the 5-year mortality and PTC recurrence were developed based on the risk factors in the training set and validated in the independent testing and validation sets.

Conclusion: The preoperative LMR was identified as an independent prognostic factor that could be incorporated into the two nomograms with other risk factors to predict overall survival and PTC-free survival for individual patients.

Keywords: lymphocyte-to-monocyte ratio, papillary thyroid carcinoma, lymph node metastasis, recurrence

\section{Introduction}

The incidence of papillary thyroid carcinoma (PTC) continues to increase worldwide. ${ }^{1}$ PTC exhibits a broad range of clinical behaviors ranging from indolent tumors with low mortality in most cases to very aggressive malignancies. ${ }^{2}$ According to the 2015 American Thyroid Association (ATA) recurrence risk stratification, ${ }^{3}$ the postoperative recurrence rate of low-risk PTCs is less than 5\%; however, that of high-risk PTCs can reach more than $20 \%{ }^{2}$ Thyroidectomy and metastatic lymph node dissection remain the main curative treatments for PTCs; for high-risk PTC cases, postoperative 131-I is an important possible adjuvant treatment. However, the recurrence rates for high-risk patients are high and variable, largely due to locoregional recurrence, metastases or radioiodine (RAI) refractoriness. Therefore, an urgent precise classification of highrisk PTC is needed to better predict patient outcomes and adjuvant RAI responses.

Recently, certain systemic inflammatory markers, such as the lymphocyte-to-monocyte ratio (LMR), have been reported as predictive and prognostic factors for human 
cancers. ${ }^{4-6}$ However, the prognostic predictive role of the LMR remains unclear in PTCs, especially in high-risk PTCs with increased risk of recurrence/metastasis or mortality. In our previous study, ${ }^{7}$ we reported that a lower preoperative LMR was related to a higher rate of lymph node metastasis (LNM) in medullary thyroid carcinoma (MTC). Therefore, the incorporation of the LMR into a prognostic model might add prognostic value to further stratify and better manage high-risk patients with different prognoses.

According to the 2015 ATA guidelines, ${ }^{3}$ high-risk PTC was defined as cases meeting any of the following characteristics: gross extrathyroid extension (GEE), distant metastases, and LNM for which the largest tumor diameter is at least $3 \mathrm{~cm}^{2}$. The recurrence/metastasis rate ranged from $20 \%$ to $72 \%$. $^{3,8}$ This risk-stratification system cannot be applied to individuals due to its noncontinuous nature. Additional insights could be gained by appreciating that the risk of structural disease recurrence is a continuum of risk, as recommended in the 2015 ATA guidelines. ${ }^{3}$

In our present study, we explored the relationship of the preoperative LMR with PTC clinical characteristics and clinical outcomes and attempted to establish a nomogram based on risk factors to predict the possibility of recurrent/metastasis or mortality in high-risk PTC individuals by obtaining a specific risk value.

\section{Methods}

In our present study, we enrolled 3 independent sets of PTC patients from 2 medical hospitals in Sichuan, China. The inclusion criteria were PTCs with GEE, distant metastases or LNM for which the largest tumor diameter was at least $3 \mathrm{~cm}$. The exclusion criteria were the presence of other histological thyroid cancers, such as MTC and anaplastic thyroid cancer; additional operations; and previous other cancers. Based on these criteria, 224 cases in West China Hospital (Wuhou, Sichuan) from Jan 2010 to Dec 2016 were included and then randomized into two groups using the sealed envelope method (https:/www.sealedenve lope.com/): the training and testing sets included 112 cases each. An additional 48 cases (from Dec 2012 to Dec 2016) met the inclusion and exclusion criteria from Chengdu Shang Jin Nan Fu Hospital (Pixian, Sichuan) and were set as the external validation group. This study was approved by the Clinical Research Ethics Committee of the two hospitals, and the patients or their families signed the informed consent form. The study complied with the standards set by the Declaration of Helsinki.
The GEE definition was based on the surgeon's discovery of GEE during the operation and the pathologist's subsequent designation of $\mathrm{pT} 4 ;^{9}$ distant metastases were evaluated mainly by imaging screening and then confirmed by $3-5 \mathrm{mCi}$ of $131-\mathrm{I}$ for scanning. The surgical protocols for PTC were mainly total or near total thyroidectomy combined with bilateral central compartment lymph node dissection; lateral compartment lymph node dissection was performed only therapeutically (when fine needle aspiration (FNA) cytology or thyroglobulin assessment proved metastasis). The details of the surgical procedure and postoperative adjuvant therapies were described in our previous study. ${ }^{10}$ Postoperative RAI therapy could be empiric (30-200 $\mathrm{mCi}$ ) for these highrisk PTC cases at 6 months post surgery. RAI refractory was defined in one of the following four ways: ${ }^{3}$ the metastatic tissue did not ever concentrate RAI; metastatic tissue lost the ability to concentrate RAI; RAI was concentrated in some lesions but not in others; and metastatic disease progressed despite significant concentration of RAI. The PTC patient lists were gathered from the two hospitals' information centers, and the clinical baseline, surgical data and postoperative recovery data were then retrospectively collected manually from the His-medical system. Based on the inclusion and exclusion criteria, follow-up of the 272 cases was performed by evaluating out-patient follow-up medical records combined with phone interviews.

All of the data were input into an Excel file and then imported into SPSS 22.0 (IBM Corporation, Armonk, USA) for statistical analysis. The association between the clinicopathological features and preoperative LMR level was evaluated by the chi-square test or Fisher's exact test. Univariate and multivariate regression analyses were used to identify independent prognostic factors in the three sets, and $P<0.05$ was defined as the criterion for variable deletion when performing backward stepwise selection. Based on the identified risk factors, a nomogram of risk factors associated with recurrence was established in $\mathrm{R}$ software studio. The predictive performance of this model was evaluated in the test and validation sets using the concordance index (C-index). Kaplan-Meier analysis was used to determine the overall survival (OS) and tumor-free survival. A log-rank test was used to compare the survival between subgroups. A two-tailed test $P$-value less than 0.05 was considered significant. 


\section{Results}

\section{PTC patient demographics and}

\section{clinicopathological characteristics}

The PTC patient baseline characteristics are summarized in Table S1. There were fewer patients of an older age $(>55)$ in the validation set than in the training and testing sets $(4.2 \%$ vs. $12.5 \%$ and $8.9 \%$, respectively). These heterogeneities might help ensure that our nomogram is universally applicable because the age-related heterogeneities and heterogeneous populations of patients may be different in different districts and conditions. ${ }^{11}$ There were no significant distribution differences between patients with or without the BRAF V600E mutation in the three sets. This result was mainly due to the later inclusion of the BRAF V600E test at Shang Jin Nan Fu Hospital than at West China Hospital.

\section{Receiver operator characteristic (ROC) curve to determine the cutoff value}

We performed ROC curve analyses of the OS to select the best LMR cutoff point, as shown in Figure $1 \mathrm{~A}$ (AUC $=0.654$, $P=0.004)$. The best and most objective LMR cutoff value for predicting OS was 4.0 with the best sensitivity (0.832) and specificity (0.441), as shown in Figure 1B (AUC $=0.637$, $P=0.010)$.

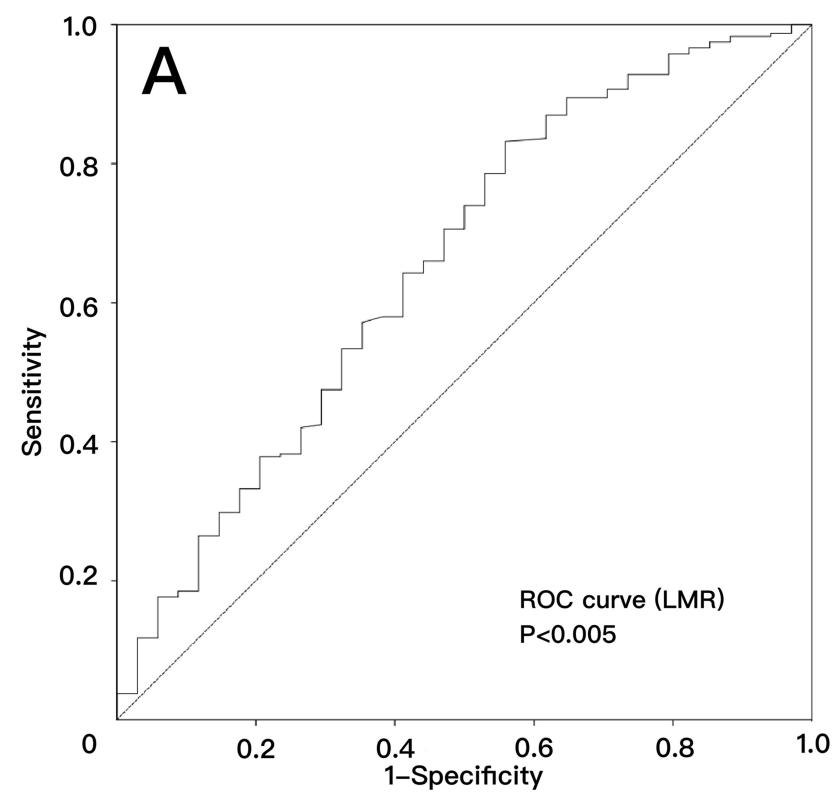

\section{Association of the preoperative LMR with PTC tumor characteristics}

The relationships between the preoperative LMR and clinical or tumor features in the training, testing and validation sets are shown in Table 1. A lower preoperative LMR correlated with a larger tumor size (all $P$-values less than 0.05 in the three sets), advanced $\mathrm{N}$ stage $(P=0.011$, $P<0.001$, and $P=0.048)$ and $\mathrm{M}$ stage $(P=0.003, P=0.024$, and $P=0.007)$, more multiple PTC cases $(P=0.001$, $P<0.001$, and $P=0.045)$, and more cases with the largest nodule larger than $3 \mathrm{~cm}(P=0.048, P=0.033$, and $P=0.023)$ in the training, testing, and validation sets, respectively. A lower LMR was also negatively correlated with the LNM number in the testing set $(P=0.009)$, whereas such a correlation was not found in the training and validation sets. This finding was mainly due to our selection of cases that were all high-risk with PTCs that were usually invasive.

\section{Correlation between the LMR and prognosis of high-risk ptcs}

Kaplan-Meier analysis was conducted to investigate the prognostic value of the preoperative LMR, and a logrank test was used to compare the overall and PTC-free survival between the low- and high-LMR groups. Patients with a high LMR gained a significant overall benefit over those with a low LMR in the training

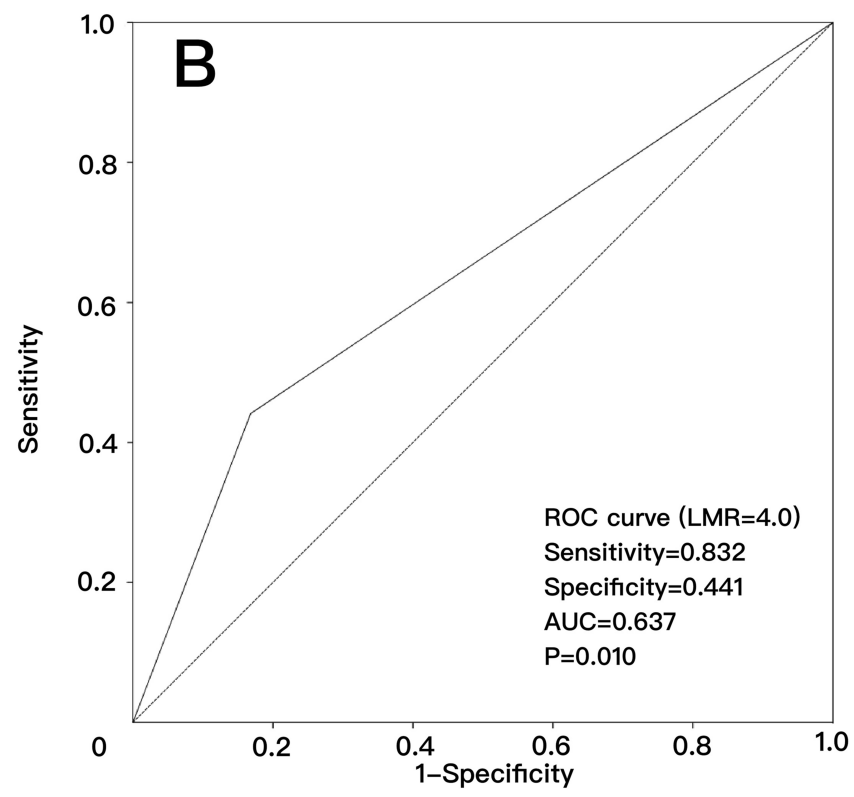

Figure I Receiver operator characteristic curve analysis to predict overall survival for high-risk PTCs using the LMR in the pooled analysis. (A) The preoperative LMR can be used to predict overall survival $(A \cup C=0.654, P=0.004)$; $(B)$ the preoperative $L M R=4.0$ is the best cutoff to predict overall survival $(A \cup C=0.637, P=0.0$ I0). 
Table I Relationships between the preoperative LMR and clinical or tumor features in three sets of PTC patients with a high risk of recurrence. A lowser preoperative LMR correlated with a larger tumor size, advanced N stage and M stage, more multiple PTC cases, and more cases with the largest nodule larger than $3 \mathrm{~cm}$ in the 3 sets

\begin{tabular}{|c|c|c|c|c|c|c|c|c|c|}
\hline \multirow[t]{3}{*}{ Factor } & \multicolumn{3}{|c|}{ Training set } & \multicolumn{3}{|c|}{ Testing set } & \multicolumn{3}{|c|}{ Validation set } \\
\hline & \multicolumn{3}{|c|}{ Preoperative LMR } & \multicolumn{3}{|c|}{ Preoperative LMR } & \multicolumn{3}{|c|}{ Preoperative LMR } \\
\hline & Low $(<4)$ & High $(\geq 4)$ & $P$ & Low $(<4)$ & High $(\geq 4)$ & $P$ & Low $(<4)$ & High ( $\geq 4)$ & $P$ \\
\hline All patients & 19 & 93 & & 19 & 93 & & 14 & 34 & \\
\hline Age (years) & & & 0.728 & & & 0.566 & & & 0.476 \\
\hline$<45$ & 10 & 53 & & 12 & 65 & & 9 & 18 & \\
\hline$\geq 45$ & 9 & 40 & & 7 & 28 & & 5 & 16 & 0.096 \\
\hline Age (years) & & & 0.188 & & & 0.540 & & 33 & 0.096 \\
\hline$<55$ & 18 & 77 & & 18 & 84 & & 12 & 33 & \\
\hline$\geq 55$ & I & 16 & & 1 & 9 & & 2 & 1 & \\
\hline Sex & & & 0.552 & & & 0.742 & & & 0.205 \\
\hline Female & 14 & 31 & & 14 & 65 & & 12 & 23 & \\
\hline Male & 5 & 62 & & 5 & 28 & & 2 & 11 & \\
\hline BMI $\left(\mathrm{kg} / \mathrm{m}^{2}\right)$ & & & 0.059 & & & 0.358 & & & 0.195 \\
\hline$<24$ & 16 & 50 & & 13 & 53 & & 12 & 22 & \\
\hline$\geq 24$ & 3 & 43 & & 6 & 40 & & 2 & 12 & \\
\hline Largest tumor size & & & $0.002^{*}$ & & & 0.014 & & & 0.017 \\
\hline$<4 \mathrm{~cm}$ & 11 & 83 & & 12 & 87 & & 8 & 29 & \\
\hline$\geq 4 \mathrm{~cm}$ & 8 & 10 & & 7 & 6 & & 6 & 5 & \\
\hline Total tumor size & & & $0.001 *$ & & & $<0.001$ & & & 0.037 \\
\hline$<4 \mathrm{~cm}$ & 8 & 74 & & 10 & 82 & & 7 & 26 & \\
\hline$\geq 4 \mathrm{~cm}$ & 11 & 19 & & 9 & 11 & & 7 & 8 & \\
\hline Intra-thyroid PTC & & & $0.001 *$ & & & $<0.001$ & & & 0.045 \\
\hline Single & 7 & 69 & & 10 & 74 & & 8 & 31 & \\
\hline Multiple & 12 & 24 & & 9 & 19 & & 6 & 3 & \\
\hline $\mathrm{T}$ classification & & & 0.258 & & & 0.112 & & & 0.374 \\
\hline TI-T3 & 4 & 32 & & 3 & 31 & & 6 & 10 & \\
\hline $\mathrm{T} 4$ & 15 & 61 & & 16 & 62 & & 8 & 24 & \\
\hline $\mathrm{N}$ classification & & & $0.011 *$ & & & $<0.00 I^{*}$ & & & $0.048 *$ \\
\hline $\mathrm{N} 0 / \mathrm{Nla}$ & I & 27 & & 1 & 20 & & 0 & 8 & \\
\hline NIb & 18 & 66 & & 18 & 73 & & 14 & 26 & \\
\hline Distant metastasis & & & $0.003^{*}$ & & & $0.024 *$ & & & $0.007^{*}$ \\
\hline No & 11 & 75 & & 11 & 76 & & 8 & 28 & \\
\hline Yes & 8 & 18 & & 8 & 17 & & 6 & 6 & \\
\hline LNM number & & & 0.210 & & & $0.009 *$ & & & 0.102 \\
\hline$<15$ & 10 & 63 & & 8 & 68 & & 3 & 16 & \\
\hline$\geq 15$ & 9 & 30 & & 11 & 25 & & 11 & 18 & \\
\hline Largest LNM diameter & & & $0.048 *$ & & & $0.033^{*}$ & & & $0.023^{*}$ \\
\hline$<3 \mathrm{~cm}$ & 11 & 71 & & 11 & 75 & & 7 & 28 & \\
\hline$\geq 3 \mathrm{~cm}$ & 8 & 22 & & 8 & 18 & & 7 & 6 & \\
\hline
\end{tabular}

Notes: $* \boldsymbol{P}<0.05$.

Abbreviations: BMI, body mass index; PTC, papillary thyroid carcinoma; T, tumor invasion depth; N, lymph node involvement; TNM, tumor node metastasis; LNM, lymph node metastasis. 
(Figure 2A, $P=0.048$ ), testing (Figure $2 \mathrm{~B}, P=0.039$ ), validation (Figure $2 \mathrm{C}, P=0.029$ ), and combined sets (Figure $2 \mathrm{D}, P<0.001$ ). The high-LMR cases also had an increased PTC-free survival benefit in the training (Figure $3 \mathrm{~A}, P=0.042$ ), testing (Figure $3 \mathrm{~B}, P=0.014$ ), validation (Figure $3 \mathrm{C}, P=0.045$ ), and combined sets (Figure 3D, $P<0.001$ ).
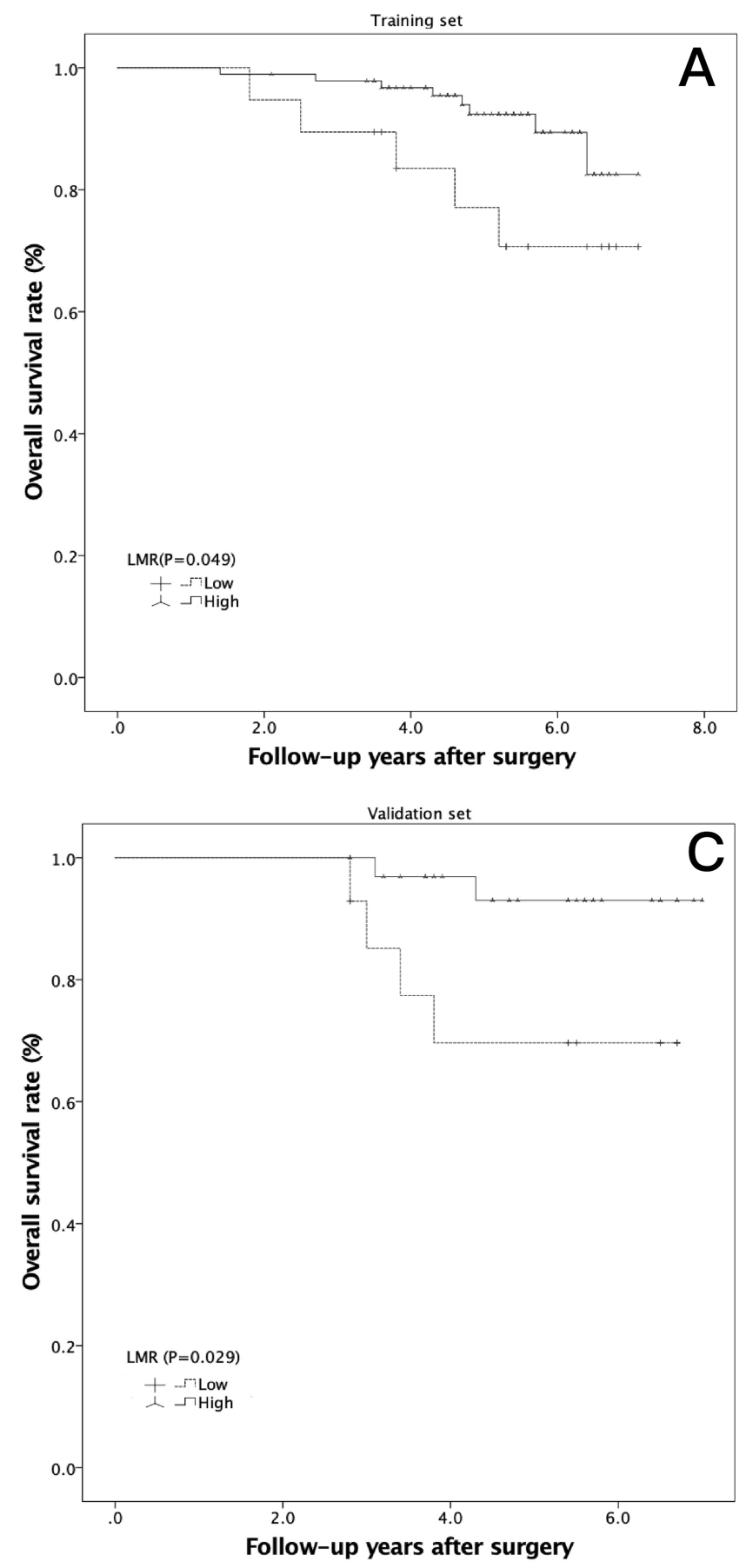

\section{Association between the LMR and postoperative RAI refractory}

Thirteen cases in the training set, 15 cases in the testing set and 7 cases in the validation set were classified as refractory to RAI after 2-6 sessions. When we compared the LMR values between the RAI refraction cases with the non-RAI refraction cases, the preoperative LMR of the RAI refraction cases was
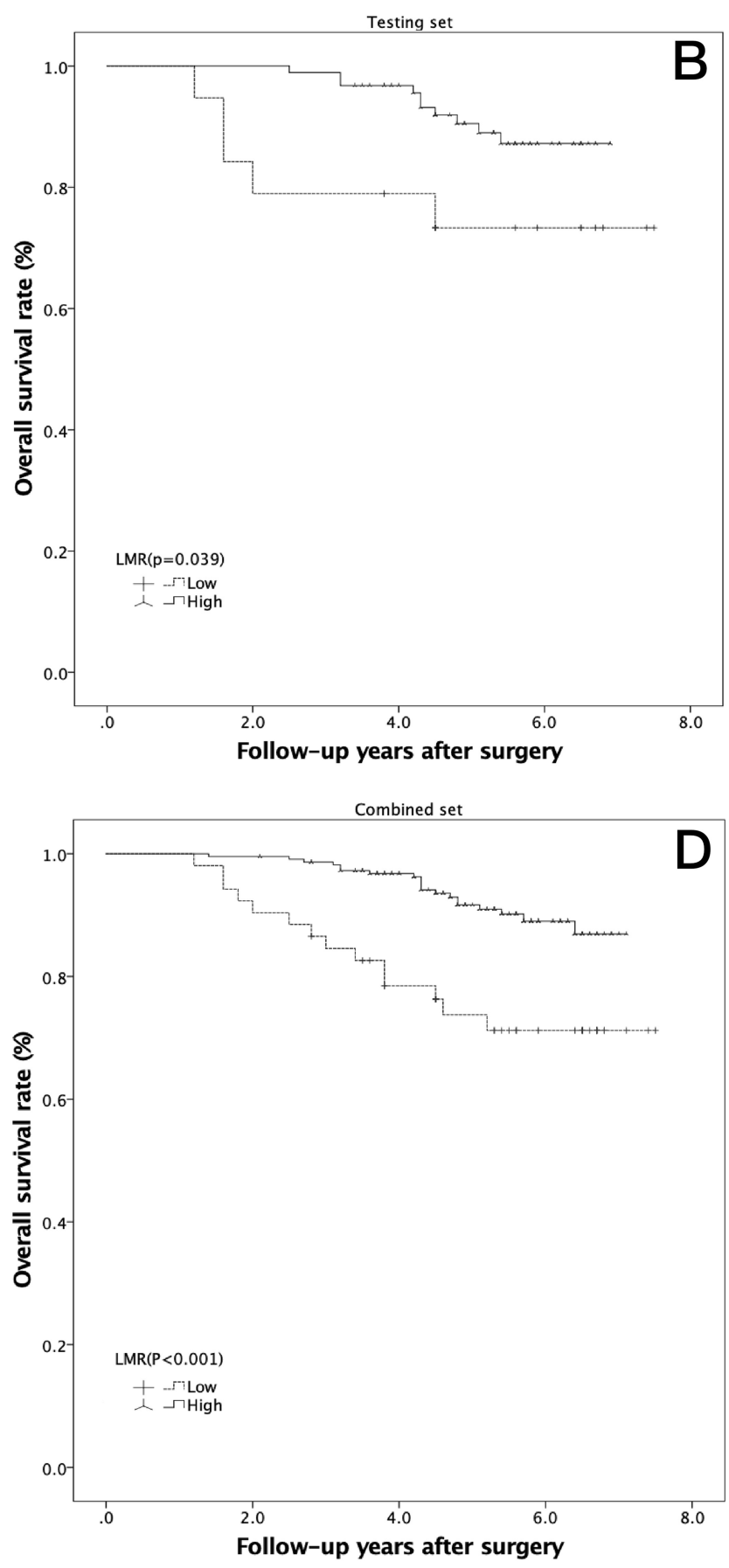

Figure 2 Kaplan-Meier analysis of overall survival in high-risk PTCs according to the LMR in 3 independent sets. $(\mathbf{A})$ Training set $(P=0.049)$; $(\mathbf{B})$ testing set $(P=0.039)$; $(\mathbf{C})$ validation set $(P=0.029)$; $(D)$ combined set $(P<0.00$ I $)$. 

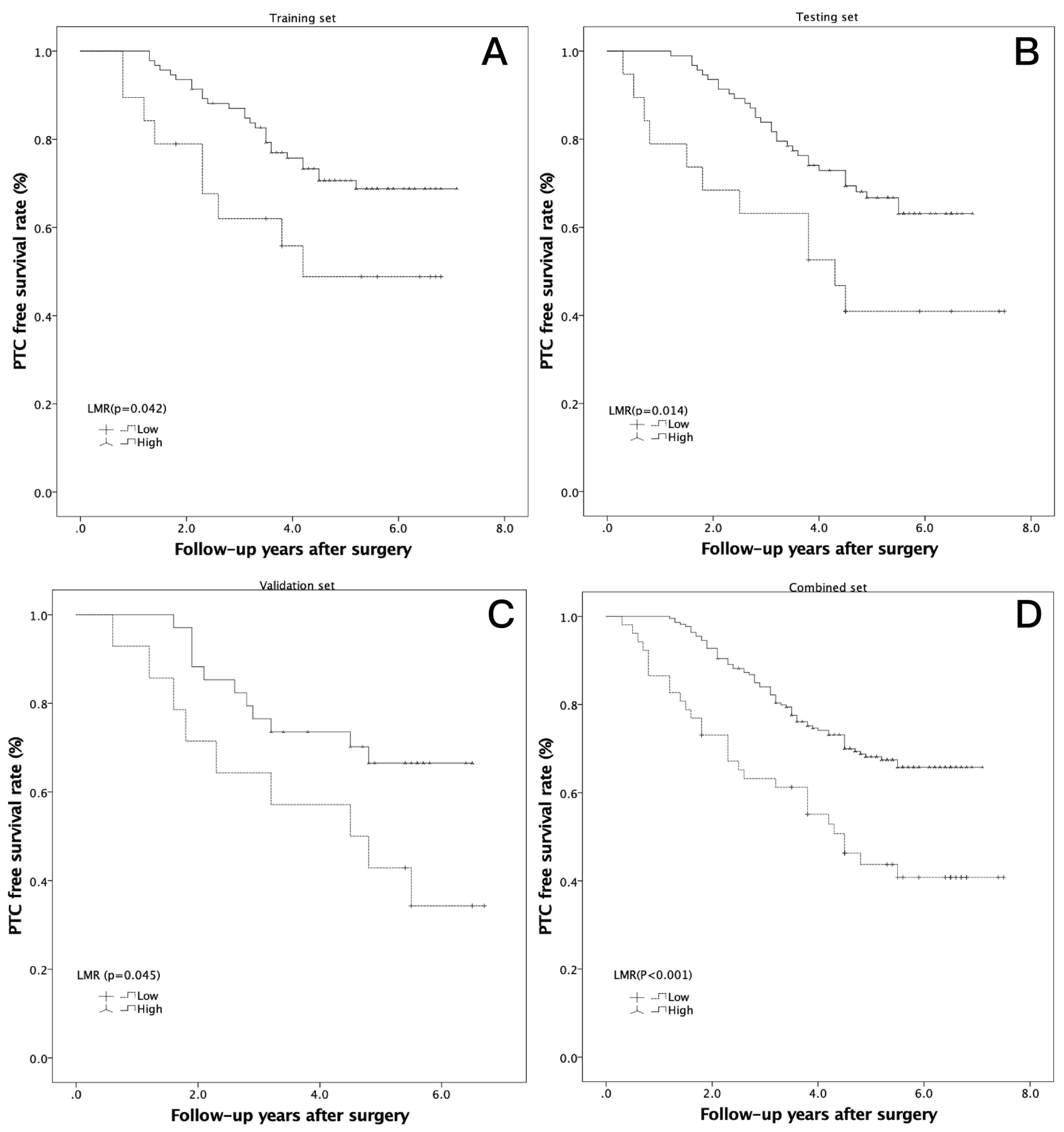

Figure 3 Kaplan-Meier analysis of PTC-free survival in high-risk PTCs according to the LMR in 3 independent sets. $(\mathbf{A})$ Training set $(P=0.042)$; $(\mathbf{B})$ testing set $(P=0.014)$; $(\mathbf{C})$ validation set $(P=0.045)$; (D) combined set $(P<0.001)$.

significantly lower than that of the non-RAI refraction cases in two sets ( $4.5 \pm 2.3$ vs. $5.9 \pm 2.0, P=0.020$ in the training set; 4.8 \pm 2.6 vs. $6.2 \pm 1.9, P=0.012$ in the testing set; and $4.1 \pm 2.7$ vs. 5.7 \pm 2.8 in validation set, which did not reach significance, $P=0.163)$. When we combined these three sets as a pooled set, this phenomenon was also observed (4.5 \pm 2.4 vs. $6.0 \pm 2.1$, $P<0.001)$.

\section{Univariate and multivariate regression analyses of overall survival and ptc-free survival}

Univariate Cox regression analysis identified clinical and tumor factors significantly associated with OS, as shown in Table 2; the largest tumor size $(P=0.037)$, total tumor 
Table 2 Univariate Cox regression analyses of overall survival in three sets of high-risk PTC patients. The largest tumor size, total tumor size, distant metastasis, LNM number, and preoperative LMR in the training set were significant. In contrast, only distant metastasis and the preoperative LMR were risk factors for OS in the testing set, and the largest tumor size, total tumor size, distant metastasis, and preoperative LMR were significant in the validation set

\begin{tabular}{|c|c|c|c|c|c|c|}
\hline \multirow[t]{3}{*}{ Factors } & \multicolumn{6}{|l|}{ Overall survival } \\
\hline & \multicolumn{2}{|l|}{ Training set } & \multicolumn{2}{|l|}{ Testing set } & \multicolumn{2}{|l|}{ Validation set } \\
\hline & HR (95\% Cl) & $\mathbf{P}$ & HR (95\% Cl) & $\mathbf{P}$ & HR (95\% Cl) & $\mathbf{P}$ \\
\hline $\begin{array}{l}\text { Age (years) } \\
\quad \leq 55 \\
>55\end{array}$ & $\begin{array}{l}\text { I (reference) } \\
2.520(0.772 \text { to } 8.230)\end{array}$ & 0.126 & $\begin{array}{l}\text { I (reference) } \\
0.683(0.090 \text { to } 5.200)\end{array}$ & 0.713 & $\begin{array}{l}\text { I (reference) } \\
4.33 \text { I (0.503 to } 37.260)\end{array}$ & 0.182 \\
\hline $\begin{array}{l}\text { Gender } \\
\text { Female } \\
\text { Male }\end{array}$ & $\begin{array}{l}\text { I (reference) } \\
2.658(0.589 \text { to II.994) }\end{array}$ & 0.204 & $\begin{array}{l}\text { I (reference) } \\
0.836 \text { (0.286 to } 2.445)\end{array}$ & 0.743 & $\begin{array}{l}\text { I (reference) } \\
0.828(0.15 \text { I to } 4.530)\end{array}$ & 0.828 \\
\hline $\begin{array}{l}\text { BMI }(\mathrm{kg} / \mathrm{m} 2) \\
\quad<24 \\
\quad \geq 24\end{array}$ & $\begin{array}{l}\text { I (reference) } \\
1.229(0.413 \text { to } 3.660)\end{array}$ & 0.711 & $\begin{array}{l}\text { I (reference) } \\
0.997 \text { (0.348 to } 2.746)\end{array}$ & 0.965 & $\begin{array}{l}\text { I (reference) } \\
0.627(0.115 \text { to } 3.426)\end{array}$ & 0.590 \\
\hline $\begin{array}{l}\text { Largest tumor size } \\
\qquad<4 \mathrm{~cm} \\
\quad \geq 4 \mathrm{~cm}\end{array}$ & $\begin{array}{l}\text { I (reference) } \\
2.280(1.072 \text { to } 10.034)\end{array}$ & $0.037^{*}$ & $\begin{array}{l}\text { I (reference) } \\
\text { I.962 (0.553 to } 6.954)\end{array}$ & 0.297 & $\begin{array}{l}\text { I (reference) } \\
8.439(1.542 \text { to } 46.201 \text { ) }\end{array}$ & $0.014 *$ \\
\hline $\begin{array}{l}\text { Total tumor size } \\
\qquad 4 \mathrm{~cm} \\
\geq 4 \mathrm{~cm}\end{array}$ & $\begin{array}{l}\text { I (reference) } \\
3.490(1.171 \text { to } 10.400)\end{array}$ & $0.025 *$ & $\begin{array}{l}\text { I (reference) } \\
\text { I. } 100 \text { (0.310 to } 3.903)\end{array}$ & 0.882 & $\begin{array}{l}\text { I (reference) } \\
5.001 \text { ( } 1.915 \text { to } 27.338 \text { ) }\end{array}$ & $0.043 *$ \\
\hline $\begin{array}{l}\text { Intra-thyroid PTC } \\
\text { Single } \\
\text { Multiple }\end{array}$ & $\begin{array}{l}\text { I (reference) } \\
\text { I.673 (0.56I to } 4.992)\end{array}$ & 0.471 & $\begin{array}{l}\text { I (reference) } \\
\text { I.99I (0.708 to } 5.600)\end{array}$ & 0.192 & $\begin{array}{l}\text { I (reference) } \\
1.688(0.800 \text { to } 5.907)\end{array}$ & 0.743 \\
\hline $\begin{array}{l}\text { T classification } \\
\text { TI-T3 } \\
\text { T4 }\end{array}$ & $\begin{array}{l}\text { I (reference) } \\
2.69 \mid(0.596 \text { to } 12.157)\end{array}$ & 0.198 & $\begin{array}{l}\text { I (reference) } \\
\text { I.878 (0.530 to } 6.657)\end{array}$ & 0.329 & $\begin{array}{l}\text { I (reference) } \\
\text { I.057 (0.193 to5.780) }\end{array}$ & 0.949 \\
\hline $\begin{array}{l}\text { N classification } \\
\text { No/NIa } \\
\text { NIb }\end{array}$ & $\begin{array}{l}\text { I (reference) } \\
4.027(0.523 \text { to } 31.033)\end{array}$ & 0.181 & $\begin{array}{l}\text { I (reference) } \\
\text { I.505 (0.340 to } 6.670)\end{array}$ & 0.590 & $\begin{array}{l}\text { I (reference) } \\
25.964(0.002 \text { to } 40.728)\end{array}$ & 0.509 \\
\hline $\begin{array}{l}\text { Distant metastasis } \\
\text { No } \\
\text { Yes }\end{array}$ & $\begin{array}{l}\text { I (reference) } \\
3.156 \text { (I.5058 to } 9.4 \mid 4)\end{array}$ & $0.039 *$ & $\begin{array}{l}\text { I (reference) } \\
\text { I } 3.537 \text { (4.27| to } 42.906)\end{array}$ & $<0.001 *$ & $\begin{array}{l}\text { I (reference) } \\
6.759 \text { (I.236 to } 36.964)\end{array}$ & $0.028^{*}$ \\
\hline $\begin{array}{l}\text { LNM number } \\
\qquad<15 \\
\quad \geq 15\end{array}$ & $\begin{array}{l}\text { I (reference) } \\
7.292(2.003 \text { to } 26.54 \mathrm{I})\end{array}$ & $0.003 *$ & $\begin{array}{l}\text { I (reference) } \\
2.049(0.742 \text { to } 5.655)\end{array}$ & 0.166 & $\begin{array}{l}\text { I (reference) } \\
3.707(0.433 \text { to } 31.749)\end{array}$ & 0.232 \\
\hline $\begin{array}{l}\text { Largest LNM diameter } \\
\qquad 3 \mathrm{~cm} \\
\quad \geq 3 \mathrm{~cm}\end{array}$ & $\begin{array}{l}\text { I (reference) } \\
2.144(0.719 \text { to } 6.391)\end{array}$ & 0.171 & $\begin{array}{l}\text { I (reference) } \\
\text { I.3।4(0.4I8 to } 4.129)\end{array}$ & 0.640 & $\begin{array}{l}\text { I (reference) } \\
\text { I.459(0.267 to } 7.972)\end{array}$ & 0.663 \\
\hline $\begin{array}{l}\text { Preoperative LMR } \\
\text { Low } \\
\text { High }\end{array}$ & $\begin{array}{l}\text { I (reference) } \\
0.340(0.110 \text { to } 0.912)\end{array}$ & $0.031 *$ & $\begin{array}{l}\text { I (reference) } \\
0.340 \text { (0.116 to } 0.997)\end{array}$ & $0.049 *$ & $\begin{array}{l}\text { I (reference) } \\
0.185(0.034 \text { to } 0.912)\end{array}$ & $0.042^{*}$ \\
\hline
\end{tabular}

Notes: $* P<0.05$.

Abbreviations: $\mathrm{HR}$, hazard ratio; $95 \% \mathrm{Cl}, 95 \%$ confidence interval; $\mathrm{T}$, tumor invasion depth; $\mathrm{N}$, lymph node involvement; LNM, lymph node metastasis. 
size $(P=0.025)$, distant metastasis $(P=0.039)$, LNM number $(P=0.003)$, and preoperative LMR $(P=0.031)$ in the training set were significant. In contrast, only distant metastasis $(P<0.001)$ and the preoperative LMR $(P=0.049)$ were risk factors for OS in the testing set, and the largest tumor size $(P=0.014)$, total tumor size $(P=0.043)$, distant metastasis $(P=0.028)$, and preoperative LMR $(P=0.042)$ were significant in the validation set. As shown in Table 3, variables demonstrating a significant effect on OS were included in the multivariate analysis. The largest tumor size $(P=0.044, P=0.032, P=0.048$ and $P=0.045$, respectively), distant metastasis $(P=0.045$, $P<0.001, P=0.050$ and $P<0.001$, respectively), and preoperative LMR $(P=0.018, P=0.019, P=0.048$ and $P=0.020$, respectively) were identified as independent prognostic factors associated with OS in the 3 sets and in the pooled analysis.

As shown in Table S2, the largest tumor size $(P=0.016$, $P=0.017$ and $P=0.045)$, distant metastasis $(P=0.031$, $P<0.001$ and $P=0.023)$, largest LNM diameter $(P=0.008$, $P=0.048$ and $P=0.011)$, and preoperative LMR $(P=0.048$, $P=0.018$ and $P=0.042$ ) were risk factors for PTC-free survival in the training, testing and validation sets, respectively, and the total tumor size $(P=0.021)$ and T classification $(P=0.013)$ were identified as risk factors in only the training set. As shown in Table S3 , variables demonstrating a significant effect on PTC-free survival were included in the multivariate analysis. The largest tumor size ( $P=0.049, P=0.038, P=0.050$ and $P=0.045)$, distant metastasis $(P=0.016, P<0.001, P=0.014$ and $P=0.002)$, largest LNM diameter $(P=0.048, \quad P=0.024, \quad P=0.034 \quad$ and $P=0.033)$, and preoperative LMR $(P=0.018, P=0.037$, $P=0.049$ and $P=0.021$ ) were identified as independent prognostic factors associated with OS in the 3 sets and in the pooled analysis, respectively.

\section{Nomogram for predicting OS and ptc- free survival}

As shown in Tables 3 and $\underline{S 3}$, all risk factors that showed significance in the training set were included in the nomogram, tested by the testing set and validated by the validation set. As shown in Figure 4, the likelihood of 5-year mortality (Figure 4A) and recurrence (Figure 4B) in individual PTC patients was easily calculated. Gathering these risk factor data, each risk factor can be used to obtain a number of points. Then, the points are added to calculate their total number. From the total-points axis, a line can be

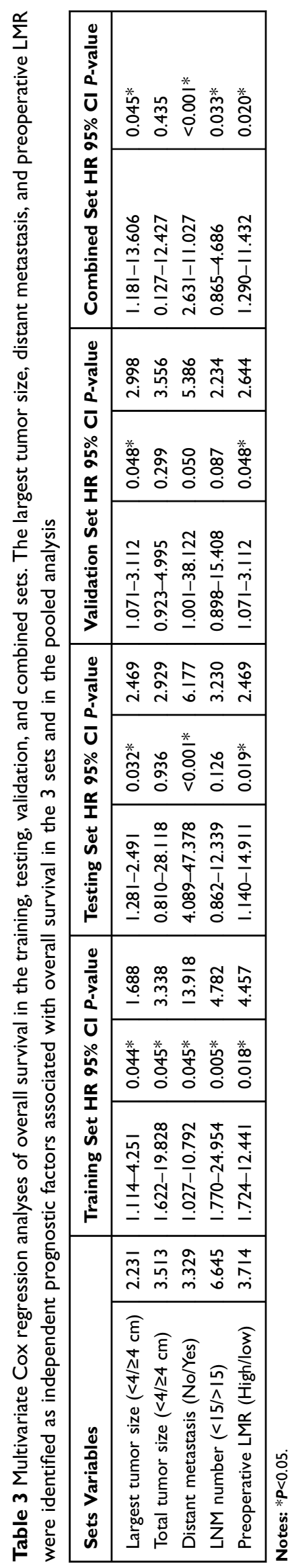




\section{A}

Points

10

50

60

70

90

Preoperative_LMR

Largest_tumor_size ( $\mathrm{mm}$ )

LNM_number

Distant_metastasis

Total Points

Risk (Risk of 5-years' mortality)

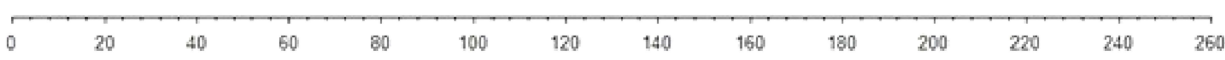

B

Points

Preoperative_LMR

Largest_tumor_size (mm)

Largest_LNM_diameter ( $\mathrm{mm}$ )

Distant_metastasis

T_stage

Total Points

Risk (Risk of 5-years' PTC recurrence)
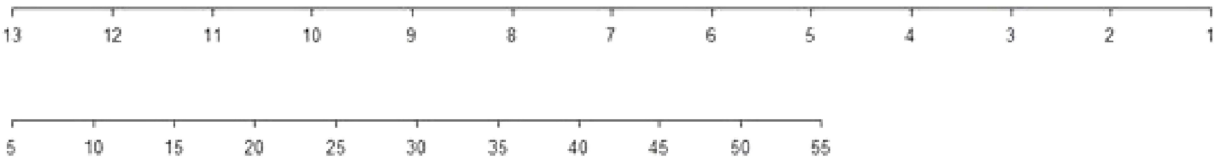

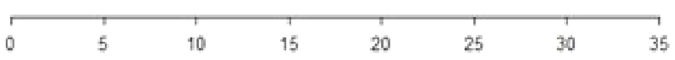

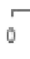

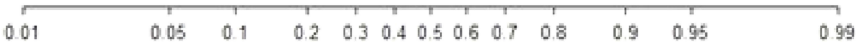

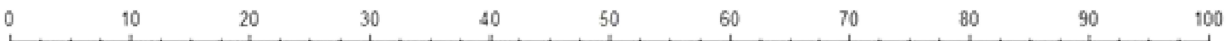

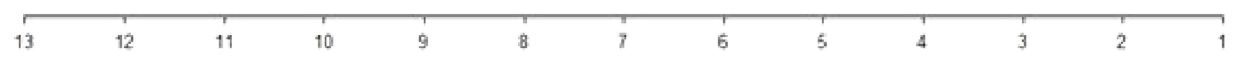

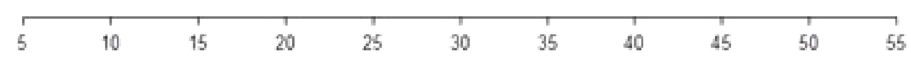

\begin{tabular}{lllllllllll}
\hline 5 & 10 & 15 & 20 & 25 & 30 & 35 & 40 & 45 & 50 & 55
\end{tabular}$$
0
$$

$\begin{array}{llll}2 & & 4 & \\ 1 & 3 & & 5\end{array}$

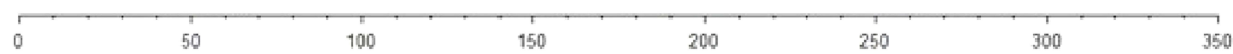

\begin{tabular}{lllllllllll}
\hline 0001 & 0.01 & 0.05 & 0.1 & 02 & 0.3040 .50 .60 .7 & 0.8 & 0.9 & 0.95 & 0.99 & 0.999
\end{tabular}

Figure 4 Nomogram for predicting high-risk PTC patient mortality (A) and PTC recurrence (B) for individual high-risk PTC patients. We can calculate the risk of early recurrence as follows. After obtaining the risk factor data, each risk factor can be used to calculate the number of points. Then, the points are added to obtain a total value. On the total-points axis, a line is drawn down to the risk axis. Then, the risk probabilities of mortality (A) and PTC recurrence (B) can be calculated for individual patients.

drawn down to the risk axis. Then, the risk of 5-year mortality and recurrence can be calculated for individual patients. In the internal (testing set) and external (validation set) validation cohorts, the C-indexes of the PTC recurrence nomogram were 0.78 (95\% CI: 0.67 to $0.86)$ and 0.77 (95\% CI: 0.64 to 0.82$)$, respectively. 
Therefore, the proposed nomograms performed well for all high-risk PTC cases.

\section{Discussion}

Our study might be considered one report in the context of several on the LMR in various human cancers; ${ }^{5,12}$ however, our present study has some potential innovations. First, to our knowledge, this is the first study on the relationship between the preoperative LMR of PTC patients and PTC features or prognosis. Second, we further stratified the high-risk PTCs according to the 2015 ATA guidelines. Third, a nomogram was developed based on the risk factors and can be used to calculate individual patients' 5-year mortality and PTC recurrence risks. Fourth, we detected that patients with a low LMR were more likely to be RAI-refractory than those with a high LMR. Finally, all of the results were tested in the internal cohort patients (testing group) and validated in the external cohort patients (validation group).

Inflammation plays a crucial role in cancer development and progression ${ }^{13}$ and could be a key determinant of outcomes in cancer patients. ${ }^{14}$ Inflammation can destroy cancer cells but also establishes a tumor microenvironment that facilitates cancer cell proliferation. ${ }^{14}$ Several inflammatory parameters have been reported, and some have been converted to ratios such as the neutrophil to lymphocyte ratio (NLR) and LMR. Lymphocytes are key immune cells, and a low lymphocyte count has been frequently observed in patients with advanced cancer and is associated with a poor OS in various cancers. ${ }^{15,16}$ Monocytes are also major immune cells and are recruited into tumors and alter the tumor microenvironment to promote cancer cell progression through immune suppression and angiogenesis. ${ }^{17,18}$ Moreover, a low ratio of peripheral lymphocytes to monocytes has been associated with advanced PTC stage features, including larger tumor sizes, advanced lymph node stages and metastases, in our analysis and is identified as a poor prognostic indicator, including lower OS and PTC-free survival rates, which is consistent with previous studies on other cancers. ${ }^{5,19}$ However, the potential mechanism by which low-LMR PTC cases were more likely to be RAI-refractory was not explained in our present study. Lymphocytes or monocytes might change the expression of the sodium/iodide symporter, which is the main mechanism of a RAI-refractory status. Future research is needed regarding this issue. The cutoff values of the LMR range greatly, from 2.0 in urothelial cancer to 5.26 in endometrial cancer, ${ }^{12}$ and there is no reference cutoff value for PTC patients; however, we detected the cutoff value by ROC curve analysis and confirmed this value in our further analysis.

Our present study included only high-risk PTCs and excluded low- and intermediate-risk cases. Two reasons contributed to this selection: first, low-risk patients have an excellent prognosis, with only $2-3 \%$ experiencing recurrence and a nearly $1 \%$ 10-year mortality rate. Intermediate-risk patients exhibit a 5-8\% recurrence rate and a $2-5 \% 10$-year mortality rate. ${ }^{3}$ These data encourage the performance of a multiple-center, large-cohort case analysis to determine the significance, and we are planning this study in the future. Second, one aim of this study was to stratify high-risk PTCs with very diverse prognoses, and our multivariate analysis and nomogram achieved this goal. With a median of 5.3 years of follow-up, the overall recurrence rate was $37.5 \%$, which was comparable to those reported in previous studies; ${ }^{20}$ Pitoia et $\mathrm{al}^{21}$ reported 171 high-risk PTC cases, with a median follow-up of 4 years and a recurrence rate of $31.0 \%$, which is slightly lower than ours. Tuttle et $\mathrm{al}^{22}$ reported a high-risk PTC patient recurrence of $68.0 \%$ with a median follow-up of 7 years. This large gap among previous studies might be caused by selection bias, and none of these studies attempted to further stratify high-risk PTCs.

This study provides some practical suggestions or tools in clinical practice for not only postoperative surveillance but also for some predictive preoperative risk factors. In our present study, we stratified high-risk PTCs using multivariate analysis and provided a certain likelihood of mortality or recurrence using nomograms. Our proposed nomograms could also be used to design a follow-up protocol after surgery and to select the proper candidates to receive potentially effective adjuvant therapy such as RAI. Furthermore, these models might facilitate communication between doctors and patients regarding the prognostic analysis and postoperative sequential therapy. ${ }^{23}$ Our study proved that a lower preoperative LMR was correlated with advanced $\mathrm{N}$ and $\mathrm{M}$ stages in the training, testing, and validation sets (all $P<0.05$ in the three sets). Our results not only help establish the follow-up protocol but also provide some useful information for preoperative surgical planning. As reports have widely discussed, the role of lymph node dissection in the treatment of PTC has been controversial, and the best indicators for routine or therapeutic neck dissection remain a subject of research. ${ }^{24}$ A low preoperative LMR may be a basis for the prophylactic dissection of lymph nodes in PTC patients. Furthermore, in addition to PTC, the inflammation 
parameter can also be used in "follicular neoplasms/suspicious for follicular neoplasms" to help the preoperative diagnosis of malignant nodules, as discussed in a large research study, ${ }^{25}$ but further studies are needed.

Our study has the following limitations: 1) as a retrospective study, the pathological variables included in the analysis were according to the original pathological diagnosis reports from the Department of Pathology and were not re-evaluated; 2) due to the routine follow-up protocol according to the guidelines and limitations of medical insurance, routine postoperative blood examinations are not recommended, and postoperative changes in the LMR could therefore not be obtained in our present study; 3 ) the nomograms need to be validated by a larger cohort at multiple centers; and 4) while the preoperative LMR was sufficiently evaluated in our study, using the postoperative LMR to monitor the tumor load also makes sense. $^{26,27}$ However, because this was a retrospective study, we paid attention to the thyroid, parathyroid, and nerve functions. We did not include the postoperative LMR into our routine examination during the follow-up period. Furthermore, the 2015 ATA guidelines did not recommend routine blood tests after surgery. Further studies on this topic are warranted.

In conclusion, we determined that a low preoperative LMR is a risk factor for an RAI-refractory status, mortality and recurrence. Furthermore, we developed two nomograms to objectively and accurately predict OS and PTCfree survival in high-risk PTC patients.

\section{Abbreviations}

ATA, American Thyroid Association; PTC, papillary thyroid carcinoma; LMR, lymphocyte-to-monocyte ratio; LNM, lymph node metastasis; MTC, medullary thyroid carcinoma; BMI, body mass index; $\mathrm{T}$, tumor invasion depth; N, lymph node involvement; TNM, tumor node metastasis; BMI, body mass index.

\section{Acknowledgment}

This study was supported by grants from the National Key R\&D Program of China (2017YFC0907504), the National Natural Science Foundation (81702646), the Sichuan Province Science and Technology Project of China (no. 2017SZ0139), the Sichuan University for Youth Fund (2017SCU11016), the Health and Family Planning Commission of Sichuan Province (17PJ296), and the Postdoctoral Sustentation Fund of Sichuan University (2017SCU12035).

\section{Author contributions}

All authors made substantial contributions to the conception and design, acquisition of data, or analysis and interpretation of data; took part in drafting the article or revising it critically for important intellectual content; gave final approval of the version to be published; and agree to be accountable for all aspects of the work.

\section{Disclosure}

The authors report no conflicts of interest in this work.

\section{References}

1. Siegel RL, Miller KD, Jemal A. Cancer statistics, 2017. CA Cancer J Clin. 2017;67:7-30. doi:10.3322/caac.21387

2. Cabanillas ME, McFadden DG, Durante C. Thyroid cancer. Lancet. 2016;388:2783-2795. doi:10.1016/S0140-6736(16)30172-6

3. Haugen BR, EK A, KC B, et al. 2015 American Thyroid Association Management Guidelines for Adult Patients with Thyroid Nodules and Differentiated Thyroid Cancer: The American Thyroid Association Guidelines Task Force on Thyroid Nodules and Differentiated Thyroid Cancer. Thyroid. 2016;26:1-133.

4. Zenan $\mathrm{H}$, Zixiong L, Zhicheng Y, et al. Clinical prognostic evaluation of immunocytes in different molecular subtypes of breast cancer. $J$ Cell Physiol. 2019234(11):20584-20602. doi:10.1002/jcp.28662

5. Chan JC, Chan DL, Diakos CI, et al. The lymphocyte-to-monocyte ratio is a superior predictor of overall survival in comparison to established biomarkers of resectable colorectal cancer. Ann Surg. 2017;265:539-546. doi:10.1097/SLA.0000000000001743

6. Ahn J, Song E, Oh H-S, et al. Low lymphocyte-to-monocyte ratios are associated with poor overall survival in anaplastic thyroid carcinoma patients. Thyroid. 2019;29:824-829. doi:10.1089/thy.2018.0684

7. Jiang $\mathrm{K}$, Lei J, Li C, et al. Comparison of the prognostic values of selected inflammation based scores in patients with medullary thyroid carcinoma: a pilot study. J Surg Oncol. 2017;116:281-287. doi:10.1002/jso.v116.3

8. van Velsen EFS, Stegenga MT, van Kemenade FJ, et al. Evaluating the 2015 American Thyroid Association Risk Stratification System in High Risk Papillary and Follicular Thyroid Cancer Patients. Thyroid. 2019;29:1073-1079. doi:10.1089/thy.2019.0053

9. Hay ID, Johnson TR, Thompson GB, Sebo TJ, Reinalda MS. Minimal extrathyroid extension in papillary thyroid carcinoma does not result in increased rates of either cause-specific mortality or postoperative tumor recurrence. Surgery. 2016;159:11-19. doi:10.10 16/j.surg.2015.05.046

10. Lei J, Zhong J, Jiang K, Li Z, Gong R, Zhu J. Skip lateral lymph node metastasis leaping over the central neck compartment in papillary thyroid carcinoma. Oncotarget. 2017;8:27022-27033. doi:10.18 632/oncotarget. 15388

11. Kitahara CM, Sosa JA. The changing incidence of thyroid cancer. Nat Rev Endocrinol. 2016;12:646-653. doi:10.1038/nrendo.2016.110

12. Nishijima TF, Muss HB, Shachar SS, Tamura K, Takamatsu Y. Prognostic value of lymphocyte-to-monocyte ratio in patients with solid tumors: a systematic review and meta-analysis. Cancer Treat Rev. 2015;41:971-978. doi:10.1016/j.ctrv.2015.10.003

13. Grivennikov SI, Greten FR, Karin M. Immunity, inflammation, and cancer. Cell. 2010;140:883-899. doi:10.1016/j.cell.2010.01.025

14. Zitvogel L, Pietrocola F, Kroemer G. Nutrition, inflammation and cancer. Nat Immunol. 2017;18:843-850. doi:10.1038/ni.3754

15. Ray-Coquard I, Cropet C, Van Glabbeke M, et al. Lymphopenia as a prognostic factor for overall survival in advanced carcinomas, sarcomas, and lymphomas. Cancer Res. 2009;69:5383-5391. doi:10.1158/ 0008-5472.CAN-08-3845 
16. Ceze N, Thibault G, Goujon G, et al. Pre-treatment lymphopenia as a prognostic biomarker in colorectal cancer patients receiving chemotherapy. Cancer Chemother Pharmacol. 2011;68:1305-1313. doi:10.1007/s00280-011-1610-3

17. Chanmee T, Ontong P, Konno K, Itano N. Tumor-associated macrophages as major players in the tumor microenvironment. Cancers (Basel). 2014;6:1670-1690. doi:10.3390/cancers6031670

18. Goswami KK, Ghosh T, Ghosh S, Sarkar M, Bose A, Baral R. Tumor promoting role of anti-tumor macrophages in tumor microenvironment. Cell Immunol. 2017;316:1-10. doi:10.1016/j.cellimm.2017.04.005

19. Gu L, Li H, Chen L, et al. Prognostic role of lymphocyte to monocyte ratio for patients with cancer: evidence from a systematic review and meta-analysis. Oncotarget. 2016;7:31926-31942. doi:10.18632/ oncotarget. 7876

20. Vaisman F, Momesso D, Bulzico DA, et al. Spontaneous remission in thyroid cancer patients after biochemical incomplete response to initial therapy. Clin Endocrinol (Oxf). 2012;77:132-138. doi:10.1111/j.13652265.2012.04342.x

21. Pitoia F, Bueno F, Urciuoli C, Abelleira E, Cross G, Tuttle RM. Outcomes of patients with differentiated thyroid cancer risk-stratified according to the American thyroid association and Latin American thyroid society risk of recurrence classification systems. Thyroid. 2013;23:1401-1407. doi:10.1089/thy.2013.0011

22. Tuttle RM, Tala H, Shah J, et al. Estimating risk of recurrence in differentiated thyroid cancer after total thyroidectomy and radioactive iodine remnant ablation: using response to therapy variables to modify the initial risk estimates predicted by the new American Thyroid Association staging system. Thyroid. 2010;20:1341-1349. doi:10.1089/thy.2010.0178
23. Li J, Zhou J, Yang P-H, et al. Nomograms for survival prediction in patients undergoing liver resection for hepatitis B virus related early stage hepatocellular carcinoma. Eur J Cancer. 2016;62:86-95. doi:10.1016/j.ejca.2016.04.011

24. Conzo G, Docimo G, Mauriello C, et al. The current status of lymph node dissection in the treatment of papillary thyroid cancer. A literature review. Clin Ter. 2013;164:e343-e346. doi:10.7417/CT.2013.1599

25. Conzo G, Calò PG, Gambardella C, et al. Controversies in the surgical management of thyroid follicular neoplasms. Retrospective analysis of 721 patients. Int J Surg. 2014;12 Suppl 1:S29-S34. doi:10.1016/j.ijsu.2014.05.013

26. Peng D, Lu J, Hu H, Li B, Ye X, Cheng N. Lymphocyte to monocyte ratio predicts resectability and early recurrence of Bismuth-Corlette type IV Hilar Cholangiocarcinoma. J Gastrointest Surg. 2019. doi:10.1007/s11605-018-04086-9

27. Jin F, Han A, Shi F, Kong L, Yu J. The postoperative neutrophil-tolymphocyte ratio and changes in this ratio predict survival after the complete resection of stage I non-small cell lung cancer. Onco Targets Ther. 2016;9:6529-6537. doi:10.2147/OTT.S117290

\section{Publish your work in this journal}

Cancer Management and Research is an international, peer-reviewed open access journal focusing on cancer research and the optimal use of preventative and integrated treatment interventions to achieve improved outcomes, enhanced survival and quality of life for the cancer patient.
The manuscript management system is completely online and includes a very quick and fair peer-review system, which is all easy to use. Visit http://www.dovepress.com/testimonials.php to read real quotes from published authors. 